\title{
Thymoma-associated paraneoplastic encephalitis (TAPE): Diagnosis and treatment of a potentially fatal condition
}

\author{
Cherie Parungo Erkmen, MD, ${ }^{\text {a }}$ Camilo E. Fadul, MD, ${ }^{\mathrm{b}}$ Josep Dalmau, MD, PhD, ${ }^{\mathrm{c}}$ and Kadir Erkmen, MD, ${ }^{\mathrm{d}}$ \\ Lebanon, NH, and Philadelphia, $\mathrm{Pa}$
}

Up to $50 \%$ of patients with thymoma have paraneoplastic neurologic syndromes, the most common being myasthenia gravis. ${ }^{1}$ There are rare case reports of thymoma-associated paraneoplastic limbic or extralimbic encephalitis that can lead to progressive neurologic decline and death without treatment. We report a case of a malignant thymoma presenting as paraneoplastic encephalitis and review the clinical characteristics, treatments used, and outcome of 28 patients with this disorder published according to a PubMed and MEDLINE search (1950-2010).

\section{CLINICAL SUMMARY}

A previously asymptomatic 61-year-old woman had generalized intractable seizures. Brain magnetic resonance imaging (MRI) showed bilateral foci of cortical and subcortical nonenhancing signal abnormalities on $\mathrm{T}_{2}$-weighted images (Figure 1, A). Chest computed tomography (CT) revealed a large anterior mediastinal mass (Figure $1, C$ ) that, on biopsy, showed malignant thymoma. Analysis of cerebrospinal fluid detected a predominantly lymphocytic pleocytosis and positive LGI1 antibodies (previously known as voltage-gated potassium channel antibodies). ${ }^{2}$

The patient received anticonvulsants, human intravenous immunoglobulin G (IgG) (400 g/kg daily), and corticosteroids (methylprednisolone $1 \mathrm{gm}$ daily) for 5 days plus a prednisone taper with resolution of neurologic symptoms. Four weeks later, the patient underwent complete, en bloc resection of the mediastinal mass that was invading through the pericardium (type B3, World Health Organization classification, Masaoka stage III). She received adjuvant radiation therapy (5040 cGy) owing to suspected invasion into the pericardial space. Four weeks later, brain MRI showed

\footnotetext{
From the Division of Cardiothoracic Surgery, ${ }^{\mathrm{a}}$ Section of Hematology/Oncology, ${ }^{\mathrm{b}}$ and Division of Neurosurgery, Dartmouth-Hitchcock Medical Center, Norris Cotton Cancer Center, ${ }^{\mathrm{d}}$ Lebanon, $\mathrm{NH}$; and the Division of Neuro-oncology, University of Pennsylvania School of Medicine, ${ }^{\mathrm{c}}$ Philadelphia, Pa.

This work was funded through the Division of Cardiothoracic Surgery, DartmouthHitchcock Medical Center.

Disclosures: Authors have nothing to disclose with regard to commercial support. Received for publication June 10, 2010; revisions received Sept 22, 2010; accepted for publication Oct 17, 2010; available ahead of print Dec 16, 2010.

Address for reprints: Cherie Parungo Erkmen, MD, Division of Cardiothoracic Surgery, Dartmouth-Hitchcock Medical Center, One Medical Center Dr, Lebanon, NH 03756-0001 (E-mail: Cherie.P.Erkmen@Hitchcock.org).

J Thorac Cardiovasc Surg 2011;141:e17-20

$0022-5223 / \$ 36.00$

Copyright (c) 2011 by The American Association for Thoracic Surgery doi:10.1016/j.jtcvs.2010.10.022
}
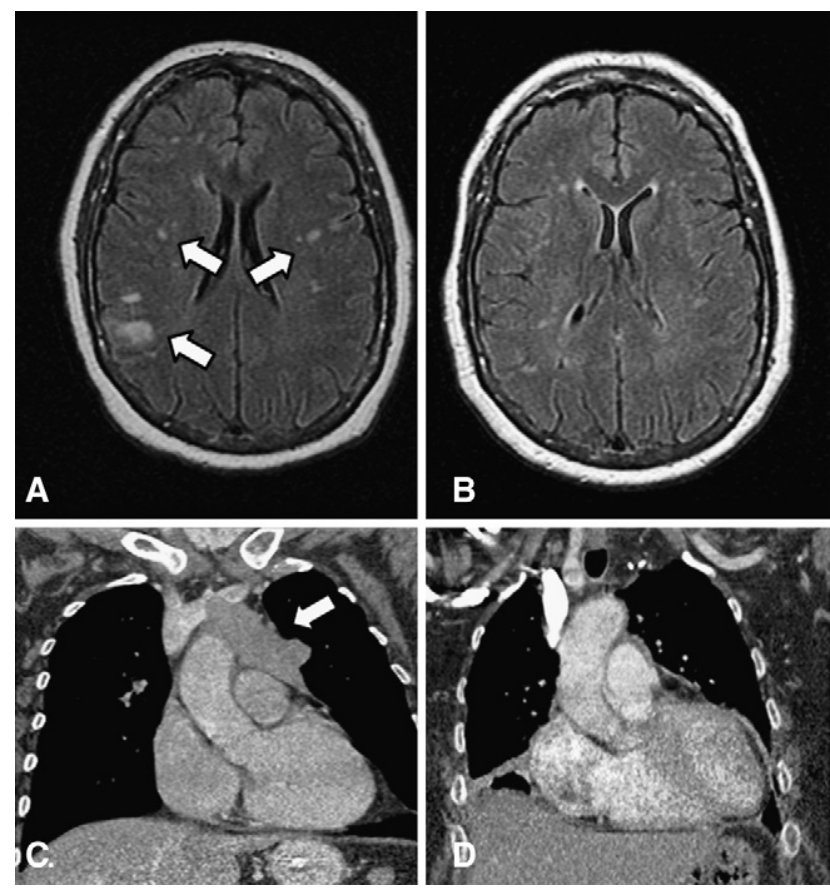

FIGURE 1. A, MRI of the head at presentation. White arrows show some of the bilateral lesions. B, MRI of the head 3 months later, after immunotherapy and surgery C, Coronal CT of the chest showing malignant thymoma invading the innominate vein. D, Coronal CT of the chest 6 month after surgery showing no residual disease. MRI, Magnetic resonance image; $C T$, computed tomogram.

decrease in size of the brain lesions (Figure 1,B). Two years after the operation, the patient has no evidence of tumor recurrence (Figure 1,D) or neurologic impairment.

\section{DISCUSSION}

Since 1988, when the first case of paraneoplastic encephalitis in the setting of thymic cancer was reported, 28 cases of thymoma-associated paraneoplastic encephalitis (TAPE) have been described (Table 1). The encephalitis can be confined to the limbic system or multifocal and extralimbic, as in our case. In most patients $(82 \%)$, neurologic manifestations include memory loss, confusion, and seizures. Seventy percent exhibited bilateral foci of nonenhancing hyperintensity on brain MRI, some appearing weeks after onset of symptoms. The differential diagnosis includes metastases, primary brain tumors, and infection. The presence of a mediastinal mass and pathologic confirmation of 
TABLE 1. Clinical features of thymoma-associated paraneoplastic encephalitis

\begin{tabular}{|c|c|c|c|c|c|c|c|c|}
\hline Author & Age/sex & Symptoms & Stage & MRI, $T_{2}$-weighted & Pleocytosis & $\begin{array}{c}\text { Antineuronal } \\
\text { antibodies }\end{array}$ & Treatment & Outcome \\
\hline $\begin{array}{r}\text { McArdle } \\
\text { (1988) }\end{array}$ & $41 / M$ & $\begin{array}{l}\text { Confusion, memory } \\
\text { deficit, } \\
\text { hallucinations }\end{array}$ & III & & No & $\begin{array}{l}\text { ANA (serum) } \\
\text { oligoclonal } \\
\text { IgG bands (CSF) }\end{array}$ & $\begin{array}{l}\text { Partial thymectomy, } \\
\text { radiation, } \\
\text { corticosteroids }\end{array}$ & Death (1 mo) \\
\hline $\begin{array}{l}\text { Ingenito } \\
\text { (1990) }\end{array}$ & $59 / \mathrm{M}$ & $\begin{array}{l}\text { Confusion, memory } \\
\text { deficit, } \\
\text { hypersomnolence }\end{array}$ & I & Normal & $13 \mathrm{WBC}$ & & Corticosteroids & Death $(51 \mathrm{~d})$ \\
\hline $\begin{array}{c}\text { Cunningham } \\
\text { (1994) }\end{array}$ & $56 / \mathrm{F}$ & $\begin{array}{l}\text { Confusion, memory } \\
\text { deficit, emotional } \\
\text { lability }\end{array}$ & IVA & $\begin{array}{l}\text { Initially normal, } \\
\text { subsequent left } \\
\text { temporal lobe lesions }\end{array}$ & & & $\begin{array}{l}\text { Thymectomy, radiation, } \\
\text { chemotherapy }\end{array}$ & $\begin{array}{l}\text { No residual } \\
\text { symptoms }\end{array}$ \\
\hline $\begin{array}{l}\text { Antoine } \\
\quad(1995)\end{array}$ & $58 / \mathrm{F}$ & $\begin{array}{l}\text { Visual and auditory } \\
\text { hallucinations, } \\
\text { confusion }\end{array}$ & III & $\begin{array}{l}\text { Hyperintensity in right } \\
\text { hippocampus }\end{array}$ & Yes & $\begin{array}{l}\text { Antineuronal } \\
\text { cytoplasmic } \\
\text { (serum and CSF) }\end{array}$ & Thymectomy, radiation & $\begin{array}{l}\text { No residual } \\
\text { symptoms }\end{array}$ \\
\hline $\begin{array}{l}\text { Antoine } \\
\quad(1995)\end{array}$ & $67 / F$ & $\begin{array}{l}\text { Confusion, agitation, } \\
\text { memory deficit, } \\
\text { hallucinations }\end{array}$ & II & $\begin{array}{l}\text { Hyperintensity } \\
\text { on right }\end{array}$ & No & $\begin{array}{l}\text { Antineuronal nuclear } \\
\text { (serum only, CSF } \\
\text { did not react) }\end{array}$ & Thymectomy, radiation & $\begin{array}{l}\text { Mild memory } \\
\text { deficit }\end{array}$ \\
\hline $\begin{array}{l}\text { Evoli } \\
\qquad(1999)\end{array}$ & $32 / \mathrm{M}$ & $\begin{array}{l}\text { Confusion, memory } \\
\text { deficit, seizures }\end{array}$ & III & $\begin{array}{l}\text { Hyperintensity and } \\
\text { edema of bilateral } \\
\text { hippocampi }\end{array}$ & $25 \mathrm{WBC}$ & & $\begin{array}{l}\text { Thymectomy, radiation, } \\
\text { chemotherapy, } \\
\text { corticosteroids, } \\
\text { plasmapheresis }\end{array}$ & $\begin{array}{l}\text { Mild memory deficit, } \\
\text { dependent on } \\
\text { corticosteroids }\end{array}$ \\
\hline $\begin{array}{r}\text { Rickman } \\
(2000)\end{array}$ & $55 / \mathrm{M}$ & $\begin{array}{r}\text { Malaise, dysarthria, } \\
\text { aphasia, seizures }\end{array}$ & II* & $\begin{array}{l}\text { Bilateral } \\
\text { hyperintensity }\end{array}$ & Yes & $\begin{array}{l}\text { AChR, CRMP5 } \\
\text { (serum) }\end{array}$ & $\begin{array}{c}\text { Partial thymectomy, } \\
\text { corticosteroids, } \\
\text { plasmapheresis }\end{array}$ & $\begin{array}{l}\text { Residual } \\
\text { symptoms }\end{array}$ \\
\hline $\begin{array}{l}\text { D'Avino } \\
\text { (2001) }\end{array}$ & $61 / \mathrm{M}$ & $\begin{array}{l}\text { Confusion, memory } \\
\text { deficit, irritable, } \\
\text { hallucinations }\end{array}$ & III & Normal & & $\begin{array}{l}\text { Antibody reactivity } \\
\text { to Perkinje cells } \\
\text { (serum and CSF) }\end{array}$ & Thymectomy, radiation & $\begin{array}{l}\text { Mild memory } \\
\text { deficit }\end{array}$ \\
\hline $\begin{array}{l}\text { Fujii } \\
\qquad(2001)\end{array}$ & $55 / \mathrm{M}$ & $\begin{array}{l}\text { Confusion, memory } \\
\text { deficit, irritable, } \\
\text { mania, seizures }\end{array}$ & $I^{*}$ & $\begin{array}{l}\text { Bilateral high } \\
\text { intensity }\end{array}$ & No & $\begin{array}{l}\text { At autopsy, } \\
\text { hippocampus } \\
\text { showing } \\
\text { anti-human IgG }\end{array}$ & Thymectomy & Death (4 mo) \\
\hline $\begin{array}{l}\text { Vernino } \\
\quad(2002)\end{array}$ & $66 / \mathrm{F}$ & $\begin{array}{l}\text { Hoarseness, } \\
\text { unilateral } \\
\text { loss of hearing, } \\
\text { areflexia }\end{array}$ & III & & & $\begin{array}{l}\text { ANNA-1, VGCC } \\
\text { (serum) }\end{array}$ & $\begin{array}{l}\text { Partial thymectomy, } \\
\text { Chemotherapy }\end{array}$ & Death (7 mo) \\
\hline $\begin{array}{l}\text { Vernino } \\
\quad(2002)\end{array}$ & $34 / \mathrm{M}$ & $\begin{array}{l}\text { Dysarthria, } \\
\text { dysphagia }\end{array}$ & II & Normal & $10 \mathrm{WBC}$ & $\begin{array}{l}\text { AChR, ANNA-1 } \\
\text { (serum) }\end{array}$ & $\begin{array}{l}\text { Thymectomy, } \\
\text { corticosteroids, } \\
\text { plasmapheresis }\end{array}$ & $\begin{array}{l}\text { Residual symptoms, } \\
\text { memory deficit, } \\
\text { labile personality }\end{array}$ \\
\hline $\begin{array}{l}\text { Vernino } \\
\quad(2002)\end{array}$ & $34 / \mathrm{F}$ & $\begin{array}{l}\text { Vertigo, tinnitus, } \\
\text { vomiting }\end{array}$ & I & $\begin{array}{l}\text { Normal, } 2.5 \text { mo later } \\
\text { bilateral cortical } \\
\text { lesions }\end{array}$ & & $\begin{array}{l}\text { ANNA-1, VGKC } \\
\quad \text { (serum) }\end{array}$ & Thymectomy & $\begin{array}{l}\text { Residual symptoms, } \\
\text { fixed right pupil }\end{array}$ \\
\hline $\begin{array}{l}\text { Vernino } \\
\quad(2002)\end{array}$ & $39 / \mathrm{F}$ & $\begin{array}{l}\text { Dysphagia, } \\
\text { weakness }\end{array}$ & III & Normal & & $\begin{array}{l}\text { ACHR, ANNA-1, } \\
\text { CRMP-5 (serum) }\end{array}$ & $\begin{array}{l}\text { Partial thymectomy, } \\
\text { radiation, } \\
\text { chemotherapy, } \\
\text { corticosteroids, } \\
\text { plasmapheresis }\end{array}$ & Not reported \\
\hline $\begin{array}{l}\text { Ances } \\
\text { (2005) }\end{array}$ & $44 / \mathrm{F}$ & $\begin{array}{l}\text { Confusion, memory } \\
\text { deficit, } \\
\text { combativeness }\end{array}$ & II & $\begin{array}{l}\text { Initially normal but } \\
5 \text { d later bilateral } \\
\text { temporal, cerebellar } \\
\text { lesions }\end{array}$ & Yes & $\begin{array}{l}\text { Neuropil reactivity } \\
\quad \text { (serum and CSF) }\end{array}$ & $\begin{array}{l}\text { Thymectomy, } \\
\text { corticosteroids, IgG }\end{array}$ & $\begin{array}{l}\text { No residual } \\
\text { symptoms }\end{array}$ \\
\hline $\begin{array}{l}\text { Ances } \\
\text { (2005) }\end{array}$ & $38 / \mathrm{M}$ & $\begin{array}{l}\text { Confusion, memory } \\
\text { deficit, agitation, } \\
\text { seizures }\end{array}$ & III & $\begin{array}{l}\text { Bilateral } \\
\text { nonenhancing } \\
\text { lesions }\end{array}$ & $7 \mathrm{WBC}$ & $\begin{array}{l}\text { Neuropil and GAD } \\
\text { (serum and CSF) }\end{array}$ & $\begin{array}{l}\text { Thymectomy, } \\
\text { corticosteroids, } \\
\text { IgG, plasmapheresis }\end{array}$ & $\begin{array}{l}\text { Memory deficit, } \\
\text { gait difficulty, } \\
\text { dependent on } \\
\text { corticosteroids }\end{array}$ \\
\hline $\begin{array}{r}\text { Chaudhry } \\
\text { (2007) }\end{array}$ & $62 / \mathrm{F}$ & $\begin{array}{l}\text { Memory deficit, } \\
\text { cognitive } \\
\text { impairment }\end{array}$ & IVA & $\begin{array}{l}\text { Bilateral hipocampal } \\
\text { hyperintensity }\end{array}$ & & & $\begin{array}{l}\text { Thymectomy, } \\
\text { chemotherapy, } \\
\text { rituimab monoclonal } \\
\text { antibody, plasmapheresis }\end{array}$ & $\begin{array}{l}\text { Progressive } \\
\quad \text { cognitive deficit }\end{array}$ \\
\hline
\end{tabular}




\begin{tabular}{|c|c|c|c|c|c|c|c|c|}
\hline Author & Age/sex & Symptoms & Stage & MRI, $\mathbf{T}_{2}$-weighted & Pleocytosis & $\begin{array}{c}\text { Antineuronal } \\
\text { antibodies }\end{array}$ & Treatment & Outcome \\
\hline $\begin{array}{l}\text { Evoli } \\
\quad(2007)\end{array}$ & & Limbic encephalitis & B2 & & & & & \\
\hline $\begin{array}{l}\text { Khella } \\
\text { (2007) }\end{array}$ & $41 / \mathrm{F}$ & $\begin{array}{l}\text { Ataxia, dysarthria, } \\
\text { dementia }\end{array}$ & I & $\begin{array}{l}\text { Bilateral } \\
\text { hyperintensity }\end{array}$ & Yes & $\begin{array}{l}\text { NMDAR/VGKC } \\
\text { (serum and CSF) }\end{array}$ & $\begin{array}{l}\text { Corticosteroids, IgG, } \\
\text { plasmapheresis }\end{array}$ & Death (31 mo) \\
\hline $\begin{array}{l}\text { Gesundheit } \\
\text { (2008) }\end{array}$ & $28 / \mathrm{M}$ & $\begin{array}{l}\text { Auditory and visual } \\
\text { hallucinations, } \\
\text { incoherent speech }\end{array}$ & B2/II & $\begin{array}{l}\text { Subtle hyperintensity } \\
\text { of temporal lobes }\end{array}$ & & & Thymectomy, IgG & $\begin{array}{l}\text { No residual } \\
\text { symptoms }\end{array}$ \\
\hline Graus (2008) & $44 / F$ & $\begin{array}{l}\text { Behavior change, } \\
\text { confusion }\end{array}$ & $I^{*}$ & Not done & Yes & $\begin{array}{l}\text { CV2 (CRMP5) } \\
\quad \text { (serum and CSF) }\end{array}$ & & Death (2 wk) \\
\hline Lai (2009) & $44 / \mathrm{F}$ & $\begin{array}{l}\text { Memory deficit, } \\
\text { confusion, seizure, } \\
\text { combativeness }\end{array}$ & II* & $\begin{array}{l}\text { Normal, } 5 \text { d later } \\
\text { increased temporal } \\
\text { lobe signal }\end{array}$ & $44 \mathrm{WBC}$ & $\begin{array}{l}\text { GluR2, ANA, dsDNA } \\
\text { cardiolipin (serum } \\
\text { and CSF) }\end{array}$ & $\begin{array}{l}\text { Thymectomy, IgG, } \\
\text { corticosteroids, } \\
\text { azathioprine }\end{array}$ & $\begin{array}{c}\text { Residual short-term } \\
\text { memory deficit }\end{array}$ \\
\hline Lai (2009) & $38 / \mathrm{M}$ & $\begin{array}{l}\text { Memory deficit, } \\
\text { confusion, seizure, } \\
\text { agitation, } \\
\text { perseveration }\end{array}$ & $\mathrm{II}^{*}$ & $\begin{array}{l}\text { Bilateral } \\
\quad \text { hyperintense } \\
\quad \text { lesions }\end{array}$ & $7 \mathrm{WBC}$ & $\begin{array}{l}\text { GluR2, GAD (serum } \\
\text { and CSF) }\end{array}$ & $\begin{array}{l}\text { Thymectomy, radiation, } \\
\text { corticosteroids, } \mathrm{IgG}, \\
\text { plasmapheresis }\end{array}$ & $\begin{array}{l}\text { Residual short-term } \\
\text { memory deficit, } \\
\text { steroid-dependen } \\
\text { muscle spasms } \\
\text { and rigidity }\end{array}$ \\
\hline Lai (2009) & $44 / \mathrm{F}$ & $\begin{array}{l}\text { Confusion, } \\
\text { behavioral } \\
\text { change, gait } \\
\text { unsteadiness, } \\
\text { progressive } \\
\text { unresponsiveness }\end{array}$ & $I^{*}$ & Normal & $15 \mathrm{WBC}$ & $\begin{array}{l}\text { GluR2, GluR1, CV2 } \\
\text { (CRMP5) (serum } \\
\text { and CSF) }\end{array}$ & $\begin{array}{l}\text { None, diagnosed } \\
\text { at autopsy }\end{array}$ & Death (24 mo) \\
\hline $\begin{array}{l}\text { Hammound } \\
\text { (2009) }\end{array}$ & $43 / \mathrm{F}$ & Seizure, confusion & B2/II & $\begin{array}{l}\text { Minimally } \\
\text { enhancing } \\
\text { bilateral lesions }\end{array}$ & Yes & $\begin{array}{l}\text { AchR, VGKC } \\
\text { (serum) }\end{array}$ & $\begin{array}{l}\text { Thymectomy, radiation, } \\
\text { chemotherapy, } \\
\text { corticosteroids, IgG }\end{array}$ & Death $(2 \mathrm{mo})$ \\
\hline $\begin{array}{l}\text { Monstad } \\
\text { (2009) }\end{array}$ & $49 / \mathrm{F}$ & Memory deficit & I & $\begin{array}{l}\text { Bilateral } \\
\text { hippocampal } \\
\text { lesions }\end{array}$ & $10 \mathrm{WBC}$ & $\begin{array}{l}\text { CRMP5, AchR } \\
\text { (serum) }\end{array}$ & $\begin{array}{l}\text { Thymectomy, } \\
\text { corticosteroids, } \\
\text { plasmapheresis }\end{array}$ & Memory deficit \\
\hline $\begin{array}{l}\text { Rizzardi } \\
\text { (2009) }\end{array}$ & $55 / \mathrm{F}$ & Seizure, aphasia & $\mathrm{B} / \mathrm{II}$ & $\begin{array}{l}\text { Multiple, bilateral lesions } \\
\text { of insular, parietal, } \\
\text { and temporal lobes }\end{array}$ & & $\begin{array}{l}\text { Normal autoimmune } \\
\text { serologic exam }\end{array}$ & $\begin{array}{l}\text { Thymectomy, } \\
\text { radiation } \\
\text { therapy }\end{array}$ & $\begin{array}{l}\text { No residual } \\
\text { symptoms and } \\
\text { disease free }\end{array}$ \\
\hline $\begin{array}{l}\text { Werry } \\
\text { (2009) }\end{array}$ & $32 / \mathrm{M}$ & $\begin{array}{l}\text { Vertigo, diplopia, } \\
\text { ataxia, myoclonic } \\
\text { jerking }\end{array}$ & $\mathrm{B} 1 / \mathrm{I}$ & $\begin{array}{l}\text { Normal, } 1 \text { mo later } \\
\text { bilateral cortical } \\
\text { nonenhancing } \\
\text { hyperintensity }\end{array}$ & $\begin{array}{l}\text { Mild } \\
\quad \text { increase }\end{array}$ & $\begin{array}{l}\text { CV2/CRMP-5 } \\
\text { (serum) }\end{array}$ & $\begin{array}{l}\text { Thymectomy, } \\
\text { corticosteroids, } \\
\text { IgG, plasmapheresis }\end{array}$ & $\begin{array}{l}\text { Headache, fatigue, } \\
\text { and myasthenic } \\
\text { ptosis and double } \\
\text { vision }\end{array}$ \\
\hline $\begin{array}{l}\text { Erkmen } \\
\quad(2010)\end{array}$ & $61 / \mathrm{F}$ & $\begin{array}{l}\text { Seizure, memory } \\
\text { deficit }\end{array}$ & III & $\begin{array}{r}\text { Bilateral nonenhancing } \\
\text { hyperintense lesions }\end{array}$ & Yes & $\begin{array}{l}\text { VGKC, cell surface } \\
\text { antigen (CSF) }\end{array}$ & $\begin{array}{l}\text { Thymectomy, radiation, } \\
\text { corticosteroids, IgG }\end{array}$ & $\begin{array}{l}\text { No residual } \\
\text { symptoms } \\
\text { and disease free }\end{array}$ \\
\hline
\end{tabular}

$M R I$, Magnetic resonance imaging; $A N A$, antinuclear antibody protein-5; $I g G$, intravenous immunoglobulin G; $C S F$, cerebrospinal fluid; $W B C$, white blood cell count; $A C h R$, acetylcholine receptor; $C R M P 5$, collapsin response mediator; $A N N A-1$, type 1 antineuronal nuclear antibody; $V G C C$, voltage-gated calcium channel; $V G K C$, voltage-gated potassium channel; $G A D$, glutamic acid decarboxylase; $N M D A R, N$-methyl-D-aspartate receptor; $C V 2$, crossveinless-2; GluR1,2, glutamate receptors 1, 2 subunits of alpha-amino3-hydroxy-5-methyl-4-isoxazolepropionic acid receptor (AMPAR); $d s D N A$, double stranded DNA. *Suspected staging based on author's description. Thymoma being stage I; thymic carcinoma being at least stage II or B3.

thymoma would suggest a paraneoplastic syndrome. Although routine analysis of cerebrospinal fluid is nonspecific, sampling of serum and cerebrospinal fluid for antineuronal antibodies (found in $75 \%$ of patients with TAPE) will aid in establishing a paraneoplastic pathogenesis. There is little value in a brain biopsy inasmuch as it usually reveals nonspecific lymphocyte and plasma cell infiltration. The diagnosis of TAPE depends on the combination of the encephalitis syndrome, MRI findings, and detection of antineuronal autoantibodies in the setting of a thymic neoplasm.
Once the diagnosis of TAPE has been made, immediate intervention is necessary to prevent progressive neurologic decline and death. Immunotherapy with IgG and corticosteroids $^{3}$ will improve neurologic symptoms resulting from inflammatory response, but complete resection of the thymoma confers the best chance at disease-free survival from oncologic as well as paraneoplastic standpoints. Antibody titers decrease after thymectomy, thus abating ongoing inflammation and cell death. ${ }^{3}$ If corticosteroids, IgG, and thymectomy fail to improve neurologic symptoms, diminishing antineuronal antibodies with plasmapheresis 
may aid in management of these patients. The treatment of TAPE must address the immune response targeting the nervous system and the underlying thymoma.

Thirty-three percent of patients with TAPE died, all as a consequence of the nervous system compromise. The majority of these patients who died had stage I thymomas. Early Masaoka stage thymomas may produce more functional and neurotoxic autoantibodies than their malignant counterparts. ${ }^{4}$ Therefore, outcome is driven more by ongoing neurologic insult than the underlying thymic disease. Only $21 \%$ of patients with TAPE achieved symptom-free survival; all of these patients had complete resection of their thymoma.

With TAPE, it is incumbent on the clinician to make the link between thymoma and encephalitis before progressive neurologic symptoms and death ensue. The reported cases suggest a role of antineuronal autoantibody production in the pathogenesis and, therefore, in the rationale for treatment options. We believe that immunotherapy and complete resection confer the best chance of symptom-free survival.

\section{References}

1. Ances BM, Vitaliani R, Taylor RA, Liebeskind DS, Voloschin A, Houghton DJ, et al. Treatment-responsive limbic encephalitis identified by neuropil antibodies: MRI and PET correlates. Brain. 2005;128(Pt 8):1764-77.

2. Lai M, Huijbers MGM, Lancaster E, Graus F, Bataller L, Balice-Gordon R, et al. Investigation of LGI1 as the antigen in limbic encephalitis previously attributed to potassium channels: a case series. Lancet Neurol. 2010;9: 776-85.

3. Antoine JC, Honnorat J, Anterior CT, Aguera M, Absi L, Fournel P, et al. Limbic encephalitis and immunological perturbations in two patients with thymoma. $J$ Neurol Neurosurg Psychiatr. 1995;58:706-10.

4. Hartmann CA, Roth C, Minck C, Niedobitek G. Thymic carcinoma. Report of five cases and review of the literature. J Cancer Res Clin Oncol. 1990;116: 69-82.

\title{
Total correction of tetralogy of Fallot associated with rhabdomyoma in the right ventricle
}

\author{
Chuan Qin, MD, Lin Chen, MD, Ren-guo Wen, MD, and Ying-bin Xiao, MD, Chong Qing, China
}

Primary cardiac tumors are a rare disease, with a low incidence. Rhabdomyoma, a type of benign primary cardiac tumor, is the most common primary cardiac neoplasm in children and occurs almost exclusively in children. We reported a rare case of an infant with tetralogy of Fallot associated with rhabdomyoma in the right ventricle.

\section{CLINICAL SUMMARY}

An 8-month-old male infant had cyanosis and a heart murmur at birth. Physical examination revealed cyanosis, a systolic murmur at the second and third intercostal spaces left of the sternum, and a weakened pulmonary second heart sound. Transthoracic echocardiography showed typical anatomic appearance of tetralogy of Fallot and a $2 \times 2-\mathrm{cm}$ mass in the right ventricular cavity. Multiple-slice com-

From the Department of Cardiovascular Surgery, Xin Qiao Hospital, the Third Military Medical University, Chong Qing, China.

Disclosures: Authors have nothing to disclose with regard to commercial support.

Received for publication Aug 3, 2010; revisions received Sept 7, 2010; accepted for publication Oct 16, 2010; available ahead of print Dec 16, 2010.

Address for reprints: Lin Chen, MD, Department of Cardiovascular Surgery, Xin Qiao Hospital, the Third Military Medical University, Chong Qing, China (E-mail: linlin1965chenchen@yahoo.cn).

J Thorac Cardiovasc Surg 2011;141:e20-2

$0022-5223 / \$ 36.00$

Copyright (C) 2011 by The American Association for Thoracic Surgery doi:10.1016/j.jtcvs.2010.10.023 puted tomography confirmed the findings from echocardiography and revealed that the mass was likely to attach to the right side of the interventricular septum (Figure 1). An on-pump open cardiac operation was performed. Cardiopulmonary bypass was established by cannulation of the aorta and venae cavae, and systemic temperature was kept around $28^{\circ} \mathrm{C}$ for the intracardiac procedure. Cold blood cardioplegia was used to induce and maintain cardiac arrest. A longitudinal incision was made crossing the pulmonary valve annulus, and the $2 \times 2 \times 1-\mathrm{cm}$ mass was found attached to the right side of interventricular septum close to cardiac apex, surrounded by muscular trabeculae (Figure 2, A). The base of the mass was about $1.5 \times 1-\mathrm{cm}$ wide. This mass had a color similar to normal myocardium with a smooth surface and irregular shape (Figure 2, B). The cardiac mass was resected completely, with care taken to cause as little injury to the normal myocardium as possible, followed by correction of tetralogy of Fallot, including infundibulectomy, repair of ventricular septal defect, and transannular pericardial patch (Figure 2,C). This infant was successfully weaned from cardiopulmonary bypass. The mass was investigated pathologically. The immunohistochemical assays showed that stainings of actin (Figure 2, $D)$, desmin, and vimentin were strongly positive. However, staining of S-100 was negative. These histologic findings proved that this cardiac mass was a rhabdomyoma. During 